\title{
Competing values, tensions and trade-offs in management of nuclear power plants
}

\author{
Teemu Reiman ${ }^{\mathrm{a}, *}$ and Carl Rollenhagen ${ }^{\mathrm{b}}$ \\ ${ }^{a}$ VTT Technical Research Centre of Finland, P.O Box 1000, FIN-02044 VTT, Finland \\ ${ }^{\mathrm{b}}$ Royal Institute of Technology, Academy for Nuclear Safety, SE-100 44 Stockholm, Sweden
}

\begin{abstract}
The specific goal of the study is to look how tensions, competing values and trade-offs manifest in the management of nuclear power plants. Second goal is to inspect how existing frameworks, such as Competing Values Framework, can be used to model the tensions. Empirical data consists of thirty interviews that were conducted as part of a NKS study on safety culture in the Nordic nuclear branch. Eight trade-offs are identified based on a grounded theory based analysis of the interview data. The competing values and potential tensions involved in the trade-offs are discussed.
\end{abstract}

Keywords: nuclear safety, safety management, competing values, trade-offs

\section{Introduction}

The specific goal of the study is to look how tensions, competing values and trade-offs manifest in the management of nuclear power plants. A specific focus is on safety of nuclear power plants. Second goal is to inspect how existing frameworks can be used to model the tensions.

Empirical data consists of thirty interviews in Finland and Sweden that were conducted as part of a NKS (see www.nks.org) study on safety culture in the Nordic nuclear branch [8]. The interviewees in both countries were selected so that they would represent the major actors in the nuclear field, i.e. the regulators, power companies, expert organizations and waste management organizations. Most of the interviewees currently held, or had experience of, a management position. Most of the interviewees had worked in the nuclear industry for at least ten years. Interviewees all had a fair amount of experience in many different positions, including operation, maintenance, engineering and human factors related issues. Most of them had a technical basic education.

The interview questions concerned the following content areas:

- Nuclear safety as a concept
- Characteristics and differences of the nuclear industries in Finland and Sweden

- Psychological characteristics of the nuclear safety culture at the Nordic countries

- Development and current challenges of the Nordic nuclear field

Tensions or trade-offs were not thus explicitly in focus in the interview. The main aim of the interviews was to get an overview of current developments and challenges associated with Nordic nuclear safety culture. In addition to the authors, Ulf Kahlbom and Elina Pietikäinen participated in carrying out the interviews. The interview material is currently being reanalyzed from the point of view of tradeoffs and competing values. This paper presents preliminary results from the analysis.

\section{Tensions and competing values}

Cameron and Quinn [1] have proposed an approach called the Competing Values Framework (CVF) to illustrate different ways of conceptualizing effectiveness and characterize organizational cultures. CVF has been developed through empirical research on indicators of effective organizations $[1,2,9]$. According to $\mathrm{CVF}$, organizations can be viewed along

\footnotetext{
* Corresponding author. E-mail: teemu.reiman@vtt.fi.
} 
two dimensions; internal versus external processes and control versus flexibility. These two dimensions form four quadrants representing organisational culture orientations or models that reflect shared or conflicting values of organisational life: (1) human relations model or clan culture; (2) open system model or adhocracy culture; (3) internal process model or hierarchy culture; (4) rational goal model or market culture.

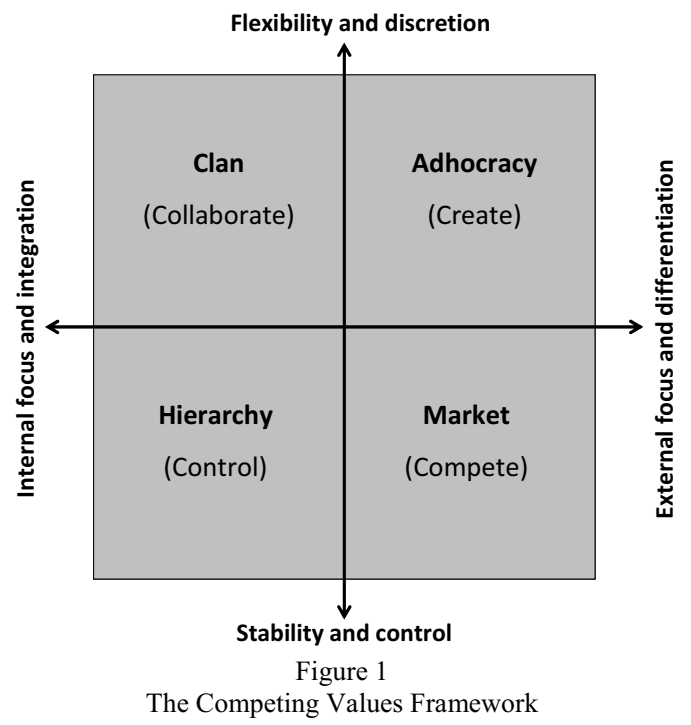

The dominant values in a clan culture are commitment, communication and development. The orientation is collaboration. Adhocracy values innovative outputs, transformation and agility. The orientation is creative. Efficiency, timeliness, consistency and uniformity are the dominant values of hierarchy culture, where the main orientation is controlling. Finally, market culture orients toward competing and values goal achievement, profitability and market share. [1] The challenge is that most organizations develop a dominant culture style; yet effective organizations need capabilities that allow them to succeed in each of the four quadrants $[1,2,9]$.

In safety critical domain, Hollnagel [4] has proposed the efficiency-thoroughness trade-off (ETTO) as being central to complex sociotechnical systems. Woods and Branlat [11] have recently discussed the fundamental tradeoffs that humans balance in sociotechnical systems. They propose five main trade-offs:

- Bounded ecology - the optimality-fragility trade-off (the system can never completely adapt to its environment and it needs to choose which criteria it seeks to optimize)
- $\quad$ Bounded cognizance - the ETTO trade off (plans are always incomplete, there is always limited time available)

- Bounded perspectivity - the acute-chronic trade off (different things seem acute when looked at different perspectives)

- Bounded responsibility - the generalistspecialist tradeoff (every system has multiple goals each of which require specialization but also integration)

- Bounded effectivity - the distributedconcentrated trade off (the system has different controllers at various places within the system)

LearnSafe EU project collected data on "perceived emerging challenges in the management of NPPs in the context of safety" by focus groups and interviews from five European countries. 593 statements were collected, 107 of which were interpreted to refer to various tensions or challenges of managing conflicting demands [5]. One generic tension concerning the focus of attention and thirteen specific tensions, e.g. economy versus safety, short-term versus long-term optimization and shared versus personal accountability, were identified.

Oedewald and Reiman [6] studied maintenance culture and maintenance core task in a Nordic nuclear power plant. They analysed how the interviewees perceive the current organisational practises identified the following, frequently addressed tensions:

- certainty vs. uncertainty about the impacts of activities

- $\quad$ specialization vs. maintaining overview

- $\quad$ situational judgment vs. generally applicable rules.

Some interviewees pointed out that there are different and conflicting ways of handling demanding situations. Other interviewees seemed to prefer one or the other way of thinking about these issues, which they did not question. For example, some people emphasized the routine nature of the work whereas some people acknowledged the uncertainties and potential hazards in everyday work. Likewise, some people considered rules as guaranteeing standardized performance whereas others emphasized that rules are always interpreted and applied according to the situation. The authors conclude that a generic issue behind all the tensions was a question about the location of responsibility for guaranteeing safety. This issue was ambiguous at the power plant, with a varying emphasis on both individual and collective responsibility. 


\section{Tensions and trade-offs in nuclear safety - grounded theory based analysis}

The analysis has so far uncovered a number of trade-offs associated with various safety related issues. The main trade-offs that we have identified concern

- autonomy versus control,

- $\quad$ understanding versus obedience,

- individual responsibility versus collective responsibility,

- awareness of the possibility of a failure versus feeling of pride and accomplishment,

- acceptable and expected events versus unexpected and unacceptable events,

- specialization versus general competence,

- centralization versus decentralization,

- negative variability versus positive variability.

\subsection{Autonomy versus control}

One of the most basic tensions in the nuclear industry is the tension between individual autonomy and system level control. This system level control manifests as technical barriers, quality control, and a strong demand for rule compliance and standardized employee behaviour.

An industry representative contemplated the challenges of work motivation and its relation to the organizational control measures:

"I guess if a person is not motivated, he turns into indifferent, and that does not go together with maintaining nuclear safety. This is also one of the small dilemmas of nuclear power. We need diverse people, but there is quite a lot, there has to be control and there is supervision. Some people might be motivated from the amount of control. Of course some people are motivated by control, but we also need those people who reflect a bit, who want to think a bit wider. So where does the border for the control go when it starts to unmotivated. So work motivation has a huge influence on everything we accomplish, and by that way also to nuclear safety. But of course the processes must be able to handle the issue that somebody is not so motivated. You cannot motivate everyone all the time, we are humans and humans have civilian life worries and other things that surely reflect to work from time to time."

Organizational functions should aim, on the one hand, at enhancing employee motivation, and on the other hand, at controlling and guaranteeing that individual human errors or negligence do not cause harm.

Maintaining high work motivation was also considered a challenge in a nuclear power plant domain. The amount of external control together with routine work was considered a threat to the work motivation. One interviewee who had a strong focus on instructions and rule compliance admitted the following:

"Well it is like this that a bit lesser quality work suffices too. I mean that one can do things very thoroughly or then in a 'that's well enough' style. Also all kinds of documentation, from the work orders and, these can be done very carelessly or then indicate clearly what has been done ... it's important for the experience feedback that there is information on what has been done."

Thus, even if the interviewees emphasized the importance of rules and instructions many acknowledged that in the end it is very much a matter of individual's sense of responsibility how well the work is done. The dilemma of autonomy and compliance is related to adherence to instructions and the responsibility of humans in general in assuring nuclear safety.

\subsection{Understanding versus obedience}

Understanding versus obedience refers to the question of whether it is enough to follow the rules or if some deeper understanding of the work is needed. One source of discussion was the amount of details in instructions: should they be so detailed that anyone can do the job by just following the instruction, or should the power companies be relying on training and a bit less detailed instructions. This tension is closely related to the conditions for giving people autonomy and it can also be considered a subcategory of the tension between autonomy and control.

Interviewees emphasized very uniformly that some kind of knowledge of the potential hazards of nuclear power is necessary for all the personnel. The following citation illustrates a common view among the respondents that especially the safety significance of one's own work has to be understood, and for that one needs some conception of the general risks of nuclear power:

"...especially [the hazards] concerning one's own work, what can in that work be such that would significantly influence more widely this .. production safety. And of course everyone should know the risks in general, of a nuclear power plant, what consequences it might have if something goes wrong. But especially on the individual level one should know at least what issues are most essential and important [in one's own work]."

It was difficult for the interviewees to define how deep knowledge of nuclear power production and its hazards the personnel need. Also, some interviewees emphasized the importance of understanding the hazards in order to increase adherence to instructions when their significance is better understood. On the 
other hand, some informants emphasized the importance of the knowledge of the hazards in order to be able to bend the instructions safely and act in situations where rules do not apply (cf. trade-off between autonomy/control and negative/positive variability).

"[one should] take into account that this is not a normal industrial work place, that in every activity the nuclear safety issues have to be noticed. ... Also at the shop floor level one would understand that why we have so many rules and why it is necessary to obey them, and why maladies have to be reported. That one does not invent some fancy work practice by oneself and think that it goes a lot faster like this. That is not approved here.

The dilemma extended also to contractors. A few interviewees mentioned struggling with the issue of how much the contractors should be educated about the basic principles of nuclear power and how much emphasis should be placed on guiding the work with strict instructions.

"One thing that has been important is that we should be able to clarify for the construction people that why we do not want certain substances, why we would not want them to smoke. Well, that we probably can never completely prevent, but these issues in general, because they are used to handle oils and such in a completely different way. And we supervise every litre that is used, and if they do not digest that there is a sense in that, then there will be gaps, where they can do anything. If they have no personal touch to the significance that one litre of thrown away oil can have."

\subsection{Individual versus collective responsibility}

The issue of responsibility was explicitly asked in the interviews. There were different emphases on the issue of responsibility; some informants emphasized the organizational responsibility whereas others emphasized the individual responsibility. One informant from the industry considered the issue ambiguously by referring simultaneously to both individual and organizational responsibility:

"Every individual should have the responsibility for those issues that the organization has assigned for him. And in addition to that one can think that the individual has a certain wider moral responsibility too. ... But in general the responsibility lies in the organization and it grows when you go upwards in the organization ... the responsibility should be perceived in relation to the power and authority that one has."

A representative from the Finnish industry contemplated the challenges of individual responsibility:

"Well, in my opinion the responsibility of the individual is to do his job as well as possible and bring forth those things that ... I wouldn't say that, this adhering to instructions is a little bit, adhering to instructions one hundred percent is not right either, if you feel that the instruction is wrong, that also has to be said. But still one should not deviate from the instructions by oneself, that's how I would put it. That is the most important thing. But this questioning and bringing forth of things and doing one's job well, that is the individual's responsibility. The organization then should not leave the individual by himself if he brings any problems forth as so on. These issues have to be then considered and thought through [collectively]."

Many interviewees also discussed the dangers of shifting personal responsibility to the organization, to instructions or to the regulators.

The following interviewee starts with an attitude that the role and responsibility are simple as the licence holder bear solely the nuclear safety responsibility but realises fast that the issue is in reality more complicated:

A: It is quite simple, I guess, the role and the responsibility... They have to do the work that is ordered from them according to the requirements that are set. And responsibility for safety is always within the licence-holder. So the licence-holder needs to be sure that the contractor does its work so that the safety level of the plant is maintained. But of course the contractor is... in order to be responsible, one has to know what he is dealing with. ...

Q: ... if the requirements aren't adequate or sufficient concerning nuclear safety, then is it still the responsibility of the subcontractor to do it according to the requirements?

A: That's a good question. In principle it probably is ... licence-holder is responsible and sets requirements that wouldn't maybe be... let's say that according to safety requirements. The contractor knows that they could do better and especially so that the safety requirement is fulfilled. Yes, a responsible contractor would of course do it so that they would guide the licence-holder like: "by the way, you don't fulfil this requirement, this has to be changed". An irresponsible contractor would maybe not do this because he would do it cheaper, the solution that fulfils the requirements that the purchaser has set. But the responsibility is within the purchaser and in Finland it is the licence-holder. And they have to know what requirements have to be fulfilled when it comes to safety. They can't land it on the contractors ...

The importance of sense of responsibility was perceived along with the many challenges associated with responsibility issues. Overall, the concept of responsibility is multidimensional and it is important to recognise its different facets. One can distinguish for example legal responsibility, moral responsibility and task responsibility (or accountability). The significance of each of these responsibilities to nuclear safety depends on the specific situation (e.g. routine work requiring adherence to instructions and task 
responsibility versus special situations requiring moral responsibility for the safety of others).

\subsection{Awareness of the possibility of failure versus feeling of pride and accomplishment}

Confidence and especially over-confidence were mentioned as one of the typical hazards of operating a smoothly running nuclear power plant. The recent events in the Nordic nuclear power industry were seen as shedding some of that over-confidence and reminding people that the hazards are real and have to be actively taken care of. One of the interviewees contemplated what the recent Nordic nuclear incidents have taught us about the nature of safety culture in the nuclear field:

"At least these events have taught us that one should never be too confident about things. This electrical disturbance at TVO last summer, it had similarities in its nature to the electrical disturbance at Forsmark, even though they were different. ... Both these cases should at least have taught that one should not think that all things are known perfectly. ... One must always think about what we do not know or understand. And the 91 Barsebäck containment sump strainer event, it was similar in that regard. One had not understood sufficiently well what kind of risks are associated with the functioning of the emergency cooling systems. ... The event demonstrated that a very essential risk factor had not been recognized. So what else has not yet been recognized? That's what is common to these events, that they teach you humility. And that's an essential thing in this field, in order to believe that safety will continuously be improved, one must be humble in that there can be something that has not been recognized. It is something that you cannot emphasize publicly too much, but it is a question of attitude."

One interviewee contemplated the potential dangerous effects for self-criticality stemming from the need to "defend" the nuclear industry against its critics:

"If you look in the mirror, nuclear power has been much questioned. The ones working in this industry believe in it - at least they are very clear about what they think. They monitor the technology and have reasonable arguments supporting that ... it is a good way for producing energy. And these points of view represent something good - but there is also a drawback: since the nuclear industry have been so much questioned for such a long time, and with so many different point of views and perspectives then it produces a situation when people tend to defend the industry from the enemies outside - the one's that are against nuclear power. This can unfortunately lead to a state when the industry fail to recognize that there are also weak points in the technology and that the whole branch, including pro- viders, operators, and regulators sometimes react in a way that would be different in another [more open] climate"

A couple of interviewees explicitly considered the trade-off between feeling proud of your accomplishments, expertise, and functioning of the plant, and the principle of continuous improvement where one starts from the assumption that there is always room for improvement and nothing is ever perfect.

\subsection{Acceptable and expected versus unacceptable and unexpected events}

Some interviewees raised the issue of design basis accidents as a source of discussion between e.g. the power companies and the regulator. A few mentioned that after the TMI accident in 1979 a new requirement for dealing with serious accidents was given. According to NRC, a design basis accident is "a postulated accident that a nuclear facility must be designed and built to withstand without loss to the systems, structures, and components necessary to ensure public health and safety". One interviewee contemplated the idea of design basis accidents:

"Of course there have earlier been these, like how to be prepared for serious reactor accidents, so this has been a big, significant... In Sweden they have been in dispute about this even earlier than in Finland. In the turn of the 1990 's, there came this preparedness for serious reactor accidents, in Olkiluoto they had to do more and also in Loviisa. There were differing opinions [between companies and authorities] at first about whether it should be done or not [because] it is a situation that is outside of design and, this serious accident, and I guess the power plants thought that if it is a situation that is outside of design, you shouldn't have to prepare for it. In Sweden they talk about these residual-risks, I mean there are other situations outside design as well that are still not prepared for, for example splitting of the reactor tank in half. This is like one thing that no-one is prepared for and can not be prepared for."

'Residual risks' refers to events that have been judged as having such an extremely small probability as an initiating event that these have not been considered in a risk analysis. The danger with residual risk is that it may transform into real risk due to changes, ageing, or increase in knowledge. Thus, even residual risk needs to be somewhat monitored instead of being labeled as residual risk forever. When discussing the philosophy of design basis accidents in the new reactors (such as the Olkiluoto 3 reactor under construction in Finland) it was noted that several accident scenarios that previously have been out-ofdesign-basis accidents have now been incorporated 
into the new design as design basis accidents. Thus, events once unexpected have now become expected. It is also interesting to note that 'expected' and 'acceptable' are not necessarily synonyms.

One challenge that is also related to specialization (see next Section) concerns the fact that people who constructed the existing plants have started to retire. In order to understand the potential disturbances of the plant its design basis and current condition need to be understood. Some interviewees expressed a worry on whether that knowledge is disappearing from the companies and the regulator.

\subsection{Specialization versus general competence}

Specialization versus general competence was raised by many interviews as an issue where tradeoffs need to be made. An interviewee described the challenges of a modernization project from the point of view of competence management and specialization:

"In the [acronym] project, the power upgrade project, quite a lot of deficiencies surfaced afterwards in both turbine and reactor side quality assurance, that components had to be sent back for repair and modified at the site. ... I think it is a sign ... if components have to be send back afterwards that there has not been enough competence [at the power plant] to supervise the process well enough, or give good enough specifications. Maybe all big components that are seldom replaced suffer from the fact that there is not enough experience at one's own organization. And maybe one of reasons is also the long supply chains. These components [at acronym project] for example have been manufactured in many places all over the world. It is clear that it is more challenging to manage the whole the more players there are. But some kind of golden middle road should be found, one cannot demand that the power company has complete deep knowledge of a component that is replaced at an interval of 20-25 years. It's impossible to ever build an organization that knows everything."

On the one hand, the interviewees seemed to perceive the trade-off between specialization and general competence, but on the other hand there were no clear solutions to the tension created by the tradeoff. One interviewee noted that:

"It is surprisingly small things that can lead to an incident ... you have to look at both large and small things"

This is easy to say but rather difficult to carry out in practice, especially considering the above mentioned challenge in perceiving things that are outside the design basis. Related to that, a few interviewees raised a worry on whether nuclear safety is consi- dered holistically enough or whether it is too fragmented into several technical disciplines and organizational levels.

\subsection{Centralization versus decentralization}

Centralization and decentralization is a typical challenge in all organizations. In nuclear field however it can have safety consequences. One interviewee told how important it was to empower the field personnel into development activities since "they know how the work is carried out and how it could be improved". Still, typical overall organizational structure in nuclear organizations is highly centralized with separation of "handicraft work" such as maintenance from more analytical engineering work and as well as from operations. 'Safety' and 'quality' also often form their distinctive disciplines inside the nuclear organization.

\subsection{Negative variability versus positive variability}

This tension dealt with the issue of how variability in human performance and technical systems was perceived. Many considered that instructions were needed since there is variability in human performance. On the other hand, there was also an opinion since instructions are not perfect either (there is variability in them too), something else is needed to compensate for that. One interviewee emphasized the extra challenges to safety culture faced in the construction phase of a nuclear power plant:

"Very often safety culture is perceived as an operation phase thing only. But it is not like that, that the design, construction and manufacturing and setting-up can be followed through by just doing things according to instructions. It's like that of course in ideal case, I guess it can be pulled off. But since we're not in an ideal world and there aren't always instructions and the instructions are sometimes wrong and plans are wrong, so then they should be intervened and addressed and brought out...

The interviewee seemed to have a conception that safety culture is needed to compensate for negative variability in the quality of instructions.

This tension also refers to the quite prevalent view of humans as the "weakest link" and human errors as the biggest threat against safety.

\section{Competing values and nuclear safety}

Table 1 summarizes the identified trade-offs. The table also presents our view of the competing values 
that underlie each end of the trade-off as well as the tensions that dealing with the trade-offs can create in the organization. When forming Table 1 it was noted that Schwartz's [10] theory of universal values can shed some more light on the nature of trade-offs. Thus, the competing values in Table 1 have been adapted partly from the CVF and partly from the universal value theory.
Many of the tensions resemble general tensions identified in the literature. For example, the Competing Values Framework has identified the dimension flexibility-control that applies to many of the here identified tensions. The universal values model also captures many of the competing values identified in this study.

Table 1. The main identified trade-offs, their associated competing values and the tensions in dealing with them

\begin{tabular}{|c|c|c|}
\hline Trade-off & Competing values & Tension \\
\hline Autonomy versus control & $\begin{array}{l}\text { Self-direction, trust, freedom, stimulation and crea- } \\
\text { tivity versus stability, conformity and con- } \\
\text { trol/dominance }\end{array}$ & $\begin{array}{l}\text { People need autonomy for motivation but excess } \\
\text { autonomy reduces predictability }\end{array}$ \\
\hline $\begin{array}{l}\text { Understanding versus } \\
\text { obedience }\end{array}$ & Self-direction versus control/dominance & $\begin{array}{l}\text { How wide and deep understanding of the work do } \\
\text { people need for the system to be able to function } \\
\text { reliably? }\end{array}$ \\
\hline $\begin{array}{l}\text { Individual versus collec- } \\
\text { tive responsibility }\end{array}$ & $\begin{array}{l}\text { Individual accountability and freedom versus collec- } \\
\text { tive accountability, cohesion and respect for tradi- } \\
\text { tion }\end{array}$ & $\begin{array}{l}\text { Organizations should avoid such collective re- } \\
\text { sponsibility where no one takes individual respon- } \\
\text { sibility for anything }\end{array}$ \\
\hline $\begin{array}{l}\text { Awareness of the possibili- } \\
\text { ty of failure versus feeling } \\
\text { of pride and accomplish- } \\
\text { ment }\end{array}$ & $\begin{array}{l}\text { Self-reflection and broadmindedness versus } \\
\text { achievement and self-respect }\end{array}$ & $\begin{array}{l}\text { How to regain adequate self-reflection without } \\
\text { losing all confidence in one's ability to succeed. }\end{array}$ \\
\hline $\begin{array}{l}\text { Unacceptable versus ac- } \\
\text { ceptable events }\end{array}$ & $\begin{array}{l}\text { Openness to change and imagination versus security } \\
\text { and orderliness }\end{array}$ & $\begin{array}{l}\text { How to prepare for the worst and yet have the } \\
\text { resources to deal with the probable }\end{array}$ \\
\hline $\begin{array}{l}\text { Specialization versus gen- } \\
\text { eral competence }\end{array}$ & $\begin{array}{l}\text { Individual accountability versus collective accoun- } \\
\text { tability }\end{array}$ & $\begin{array}{l}\text { How to assure sufficiently deep knowledge in all } \\
\text { specialty areas and yet be able to integrate that } \\
\text { knowledge into holistic understanding of the func- } \\
\text { tioning and condition of the plant }\end{array}$ \\
\hline $\begin{array}{l}\text { Centralization versus de- } \\
\text { centralization }\end{array}$ & $\begin{array}{l}\text { Control/dominance and authority versus indepen- } \\
\text { dence and individual initiative }\end{array}$ & $\begin{array}{l}\text { How to assure adequate control of the system by } \\
\text { its managers without losing the understanding of } \\
\text { the system possessed by the field level workers }\end{array}$ \\
\hline $\begin{array}{l}\text { Variability as negative } \\
\text { versus variability as posi- } \\
\text { tive }\end{array}$ & $\begin{array}{l}\text { Positive view on humans, benevolence, universalism } \\
\text { versus security and conservation }\end{array}$ & $\begin{array}{l}\text { How to dampen negative performance variability } \\
\text { and simultaneously encourage positive perfor- } \\
\text { mance variability }\end{array}$ \\
\hline
\end{tabular}

Zimmermann et al [12] point out based on their interview study that in the field of aviation "practitioners (i.e., non-experts in human factors and ergonomics, etc.) may not have a coherent, consistent, complete framework guiding how they view and understand safety. They may call up individual ideas from different paradigms or frameworks depending on the situation or the cognitive availability of the idea. There are many possible explanations for this, among them that practitioners may not have or need a coherent framework and may not even be aware when they express contradictory ideas. Or they may realize that there are frameworks but may apply different ones to different situations." In terms of effective safety management it is important to acknowledge that there are different and contradictory ways of viewing things - and then having the flexibility to move from one interpretation to other [7]. An implicit tension between competing values can then be transformed into alternate points of view on the same topic; alternate views that each shed some light on the same phenomenon. The trade-offs and the devised means of coping with them reflect as much people's beliefs as they do 'reality'.

\section{Discussion}

This study identified eight trade-offs professionals in the nuclear industry need to deal with. The interviews did not explicitly ask about trade-offs and in many cases examples of needed trade-offs emerged quite implicitly during the interviews.

The identified trade-offs were inspected in light of the Competing Values Framework. In terms of safety management, the dimension internal-external in the CVF was found to be demanding to interpret. Many of the trade-offs in the nuclear domain (and perhaps 
in other safety critical fields also) deal with autonomy/flexibility/openness/change on the one hand and individual versus collective (moral) orientation on the other hand. There might be a need for a refined framework that includes a dimension separating values related to individualism from the more collective values.

The nature of competing and conflicting value dimensions needs to be clarified in future. For example, Farjoun [3] argues that "stability and change are fundamentally interdependent - both contradictory and complementary". They are mutually enabling and constituent of each other. They represent a duality rather than dualism [3]. Further, we can differentiate stability of the outcomes (cf. High Reliability Organizations) and stability of the processes. In nuclear field the outcome should be stable (safe, reliable production), but to achieve that change, innovation and flexibility is needed.

Thus, we can argue that trade-offs and competing values are not only inevitable in any safety critical organization they are necessary for the development (and management) of the system. We need to better acknowledge and manage the trade-offs, not try to hide or remove them.

\section{Conclusions}

The study has the following implications for modeling and analysis of sociotechnical systems:

- Modelling the system at an ideal level of performance does not necessarily reveal the tensions that manifest in daily reality

- A functional model of the system can be used in identifying potential tensions but it has to be acknowledge that culture of the organization plays a major part in shaping the accepted way of dealing with tensions and making the necessary trade-offs (the belief structure of the employees)

- The competing values framework can help in analysing the competing values that the tradeoffs represent. However, the framework is quite general and it needs to be refined for use in safety critical domains. Research is needed for specifying how the model should be used in safety critical domains and what specific needs e.g. the nuclear domain sets for the model

- Competing values can be separated according to many different dimensions, such as control versus flexibility, individual versus collective morality, process/mechanisms versus outcomes [3] and internal versus external orientation [1]. Future research should clarify these dimensions and their relation (conflicting, contradictory, competing or complementary) with each other.

\section{References}

[1] K.S. Cameron and R.E. Quinn, Diagnosing and changing organizational culture. Based on the Competing Values Framework, Third Edition, Jossey-Bass, San Francisco, 2011.

[2] K.S. Cameron, R.E. Quinn, J. Degraff and A.V. Thakor, Competing values leadership. Creating value in organizations, Edward Elgar, Cheltenham, 2006.

[3] M. Farjoun, Beyond dualism: Stability and change as a duality, Academy of Management Review 35 (2010), 202-225.

[4] E. Hollnagel, The ETTO principle: efficiency-thoroughness trade-off, Ashgate, Farnhamn, 2009.

[5] J. Kettunen, T. Reiman and B. Wahlström, Safety management challenges and tensions in the European nuclear power industry. Scandinavian Journal of Management 23 (2007), 424-444.

[6] P. Oedewald and T. Reiman, Core task modelling in cultural assessment: A case study in nuclear power plant maintenance. Cognition, Technology \& Work 5 (2003), 283 - 293.

[7] T. Reiman and C. Rollenhagen, Human and organizational biases affecting the management of safety. Reliability Engineering \& System Safety 96 (2011), 1263-1274.

[8] T. Reiman, U. Kahlbom, E. Pietikäinen and C. Rollenhagen, Nuclear Safety Culture in Finland and Sweden - Developments and Challenges. NKS-239. Nordic nuclear safety research NKS, Roskilde, Denmark, 2001.

[9] R.E. Quinn, Beyond rational management: Mastering the paradoxes and competing demands of high performance. Jossey-Bass, San Francisco, 1988.

[10] Schwartz, S. H. (1992). 'Universals in the Content and Structure of Values: Theory and Empirical Tests in 20 Countries'. In M. Zanna (ed.), Advances in Experimental Social Psychology (Vol. 25). New York: Academic Press: 1-65.

[11] D. D. Woods and M. Branlat, How human adaptive systems balance fundamental trade-offs: Implications for polycentric governance architectures, in Proceedings of the Fourth Resilience Engineering Symposium, June 8-10, 2011, Sophia Antipolis, France.

[12] K. Zimmermann, J. Paries, R. Amalberti and D.H. Hummerdal, Is the aviation industry ready for resilience? Mapping human factors assumptions across the aviation sector, in: Resilience engineering in practice. A guidebook. E. Hollnagel, J. Paries, D.D. Woods and J. Wreathall, eds, Ashgate, Farnham, 2011. 